\title{
Bacillus pumilus LZP02 Promotes Rice Root Growth by Improving Carbohydrate Metabolism and Phenylpropanoid Biosynthesis
}

\author{
Hong Liu, ${ }^{1,2}$ Zhigang Wang, ${ }^{1,2,+}$ Weihui Xu, ${ }^{1,2}$ Jin Zeng, ${ }^{3}$ Lixin $\mathrm{Li}^{4}{ }^{4}$ Shenglin $\mathrm{Li}^{,}{ }^{4}$ and Zheng Gao ${ }^{5}$ \\ ${ }^{1}$ College of Life Science and Agroforestry, Qiqihar University, Qiqihar 161006, China \\ ${ }^{2}$ Heilongjiang Provincial Technology Innovation center of Agromicrobial Preparation Industrialization, Qiqihar 161006, China \\ ${ }^{3}$ State Key Laboratory of Lake Science and Environment, Nanjing Institute of Geography and Limnology, Chinese Academy of \\ Sciences, Nanjing 210000, China \\ ${ }^{4}$ Key Laboratory of Saline-alkali Vegetation Ecology Restoration, Ministry of Education, College of Life Sciences, Northeast \\ Forestry University, Harbin 150040, China \\ ${ }^{5}$ College of Life Sciences, Shandong Agricultural University, Ta'an 271000, China
}

Accepted 26 June 2020.

\begin{abstract}
Elucidation of the underlying mechanisms of plant growth promotion of rhizobacteria is very important. This study explored the mechanism by which Bacillus pumilus LZP02 promotes growth in rice roots through proteomic, transcriptomic, and metabolomic techniques. The results showed that $B$. pumilus LZP02 promoted the absorption of phosphorous, calcium, and magnesium ions by colonization of rice roots and enhanced peroxidase, catalase, superoxide dismutase, and $\mathrm{Ca}^{2+} \mathrm{Mg}^{2+}$ adenosine triphosphatase activities and chlorophyll contents in rice. The proteomic results showed that most of the differentially expressed proteins were involved in carbohydrate metabolism and that the biosynthesis of other secondary metabolites was also increased. According to RNA-seq and reverse transcriptionquantitative PCR analyses, expression of some genes involved in carbohydrate metabolism and phenylpropanoid biosynthesis was upregulated in rice roots. Regarding metabolomics, phenylpropanoid biosynthesis, starch and sucrose metabolism, the pentose phosphate pathway, and glyoxylate and dicarboxylate metabolism were increased. The results indicated that $B$. pumilus LZP02 promoted the growth of rice roots by enhancing carbohydrate metabolism and phenylpropanoid biosynthesis.
\end{abstract}

Keywords: Bacillus pumilus, biofertilizer, carbohydrate metabolism, phenylpropanoid biosynthesis, rice

Applying large amounts of chemical fertilizers to increase the yield of plants can lead to severe environmental pollution (Sureshrao et al. 2016). Biofertilizers are sustainable alternative technologies that can enhance productivity without ecological

${ }^{\dagger}$ Corresponding author: Z. Wang; wangzhigang@qqhru.edu.cn

Funding: This work was funded by the National Natural Science Foundation of China (31670375 and 31870493) and the basic research fees of universities in Heilongjiang Province, China (135309208 and 135409103).

*The $\boldsymbol{e}$-Xtra logo stands for "electronic extra" and indicates there are supplementary materials published online.

The author(s) declare no conflict of interest.

๑) 2020 The American Phytopathological Society impacts (Nayak et al. 2019). Plant growth-promoting rhizobacteria (PGPRs) colonize plant roots and have beneficial effects (Hermosa et al. 2012). Indeed, PGPRs can be used as biofertilizers, and these microorganisms play an important role in creating a favorable ecological environment in the rhizosphere, reducing the use of chemical fertilizers and pesticides, inhibiting the occurrence of pests and diseases, and ensuring the sustainable development of modern agriculture while achieving the goal of increased production (Li et al. 2020; Naik et al. 2019; Z. Wang et al. 2019b).

Rice (Oryza sativa) is one of the most important stable food crops in the world. In Asia, more than two billion people alone derive $80 \%$ of their energy needs by consuming rice (Chaudhari et al. 2018). PGPRs have been applied to rice in various forms to enhance growth and yield. For example, Burkholderia pyrrocinia BRM 32113 resulted in significant increases in growth promotion and reduced area under the disease progress curve in rice (Arriel-Elias et al. 2019). Azospirillum brasilense can colonize rice roots and promote growth (Thomas et al. 2019), and Paenibacillus polymyxa $\mathrm{Sx} 3$ significantly promoted growth and suppressed bacterial leaf blight in rice (Abdallah et al. 2019). In addition, Bacillus pumilus enhanced the tolerance of rice to a combination of $\mathrm{NaCl}$ and high-boron stress (Khan et al. 2016). The B. pumilus LZP02 strain used in the present study is a phosphate-dissolving bacterium that produces auxin and gibberellin and has the ability to synthesize iron carriers (Liu et al. 2018b; H. Wang et al. 2019). However, in-depth research on the promotion mechanism of PGPRs in the rice rhizosphere has not been carried out to date, even though elucidation of such mechanisms is extremely important and urgently needed. In particular, systematic studies of gene expression regulation combined with metabolic regulation are lacking, and the paucity of research in this area is a bottleneck that restricts the accurate design of strains, the optimization of interaction conditions, and the regulation of PGPR physiological functions.

In this study, B. pumilus LZP02 was added to the culture medium of rice plants, after which root growth was measured and colonization of rice roots was observed by scanning electron microscopy (SEM). Atomic absorption spectrometry was used to detect rice root nutrient absorption, and determination of the root catalase (CAT), peroxidase (POD), superoxide dismutase (SOD), and adenosine triphosphatase (ATPase) activities and chlorophyll contents was performed. These indicators 
were then combined with proteomic, transcriptomic, and metabolomic analyses to evaluate the mechanism underlying the effect of B. pumilus LZP02 on rice root growth.

\section{RESULTS}

Effect of B. pumilus LZP02 on the growth of rice roots.

The effect of $B$. pumilus LZP02 on the growth of rice roots was explored to determine the optimal inoculum concentration for a plant growth-promotion effect, and S-3 (inoculated B. pumilus LZP02 at $4 \times 10^{7} \mathrm{CFU} / \mathrm{ml}$ ) showed a higher effect (Fig. 1A). B. pumilus LZP02 markedly increased the number of rice fibrous roots in the $\mathrm{S}-3$ treatment (Fig. 1B and $\mathrm{C}$ ).

Compared with the control $(\mathrm{CK})(0 \mathrm{CFU} / \mathrm{ml})$, the length of S-3 roots increased by $62.9 \%$ (Fig. 2A), the shadow area increased by $63.2 \%$ (Fig. 2B), the surface area increased by $63.1 \%$ (Fig. 2C), the volume increased by $52.7 \%$ (Fig. 2D), the number of connections increased by $67.3 \%$ (Fig. 2E), the number of nodes increased by $68.2 \%$ (Fig. 2F), the number of root tips increased by $63.0 \%$ (Fig. $2 \mathrm{G}$ ), and the number of forks increased by $73.5 \%$ (Fig. $2 \mathrm{H}$ ). These results indicate that rice root growth was significantly enhanced under the S-3 condition.

The colonization of rice root by $B$. pumilus LZP02 was confirmed using 2.0- and 5.0-k magnification SEM, whereas the strain was not found on the roots of CK (Fig. 3).

\section{Effect of B. pumilus LZP02 on the physiological characteristics of rice roots.}

The effects of B. pumilus LZP02 on different physiological characteristics of rice roots are shown in Figure 4. The nitrogen, phosphorous, calcium, and magnesium contents of S-3 increased by $27.2,24.6,18.1$, and $28.3 \%$, respectively, compared with those of CK (Fig. 4A); meanwhile, the root activity increased by $39.2 \%$ (Fig. 4B), POD activity increased by $29.6 \%$ (Fig. 4C), CAT activity increased by $56.3 \%$ (Fig. 4D), and SOD activity increased by $59.1 \%$ (Fig. 4E). The contents of sulfur, sodium, iron, and manganese were not significantly changed. The contents of potassium and zinc were significantly reduced. The ATPase activity also exhibited significant changes; the $\mathrm{Na}^{+} \mathrm{K}^{+}$-ATPase of $\mathrm{S}-3$ was reduced by $76.7 \%$ compared with that of $\mathrm{CK}$, but the $\mathrm{Ca}^{2+} \mathrm{Mg}^{2+}$-ATPase of $\mathrm{S}-3$ increased by $56.3 \%$ (Fig. 4F); this result is consistent with the results for ion absorption. There were also significant changes in the chlorophyll content of rice leaves, whereby the chlorophyll a and chlorophyll b levels in S-3 increased by 42.2 and $50.8 \%$, respectively, compared with those of CK (Fig. 4G). These results indicate that S-3 significantly enhanced different activities in rice that can be related to plant growth promotion.

\section{Proteomic differences between CK and S-3.}

The total number of quantified proteins in CK and S-3 was 1,360 . The number of upregulated and downregulated proteins in S-3 was 103 and 640, respectively, adding a total of 743 differentially expressed proteins (DEPs) between CK and S-3 (Fig. 5A). To obtain a global picture of the proteomic changes, these 743 DEPs were annotated by gene ontology (GO) and Kyoto Encyclopedia of Genes and Genomes (KEGG) analyses. The results of the GO analysis showed that the mostrepresented categories were metabolic processes, cells, cell parts, cellular processes, binding, and catalytic activity, accounting for $12.96,12.70,12.68,12.38,11.60$, and $11.47 \%$ of the DEPs, respectively (Fig. 5B). A KEGG pathway analysis was then performed to investigate the main biological pathways in which these DEPs were enriched. The results of the KEGG_B analysis showed that most proteins are related to carbohydrate metabolism, which accounted for $28.41 \%$ of the
DEPs. This was followed by amino acid metabolism and translation, which accounted for 14.35 and $12.12 \%$ of the DEPs, respectively (Fig. 5C). Among the KEGG_C biological process terms, 15 had the largest number of DEPs. These terms included 13 metabolic processes, including one within biosynthesis of other secondary metabolites (phenylpropanoid biosynthesis), one within nucleotide metabolism (purine metabolism), three within amino acid metabolism (glutathione metabolism, cysteine and methionine metabolism, and glycine, serine, and threonine metabolism), and eight within carbohydrate metabolism (fructose and mannose metabolism, starch and sucrose metabolism, amino sugar and nucleotide sugar metabolism, the pentose phosphate pathway, glycolysis/gluconeogenesis, glyoxylate, and dicarboxylate metabolism, pyruvate metabolism, and the citrate cycle [trichloroacetic acid cycle]) (Fig. $5 \mathrm{D})$. These processes are related to the growth of plants.

\section{Transcriptomic differences between CK and S-3.}

Through the KEGG_C biological process based on the transcriptional analysis, the differentially expressed genes (DEGs) were mainly concentrated in fructose and mannose metabolism, starch and sucrose metabolism, amino sugar and nucleotide sugar metabolism, glyoxylate and dicarboxylate metabolism, phenylpropanoid biosynthesis, and the pentose phosphate pathway (Fig. 6A). This is consistent with the proteome data. As shown in Figure 6B, DEGs were observed among these processes $(P \leq 0.05)$. Compared with $\mathrm{CK}$, the S-3 treatment resulted in the differential expression of 14 genes. Under the S-3 treatment, most genes (Os02g0207900, Os05g0135500, Os09g0298200, Os07g0577300, XLOC_000876, Os08g0495800, Os01g0174300, Os03g0115100, Os08g0526100, Os03g0102100, Os12g0291100, Os02g0714200, and Os08g0154300) showed increased expression, whereas the expression of XLOC_029271 was reduced. Details regarding the genes are presented in Supplementary Table S1. Two DEGs (upregulated) participate in phenylpropanoid biosynthesis, three (twoo upregulated and one

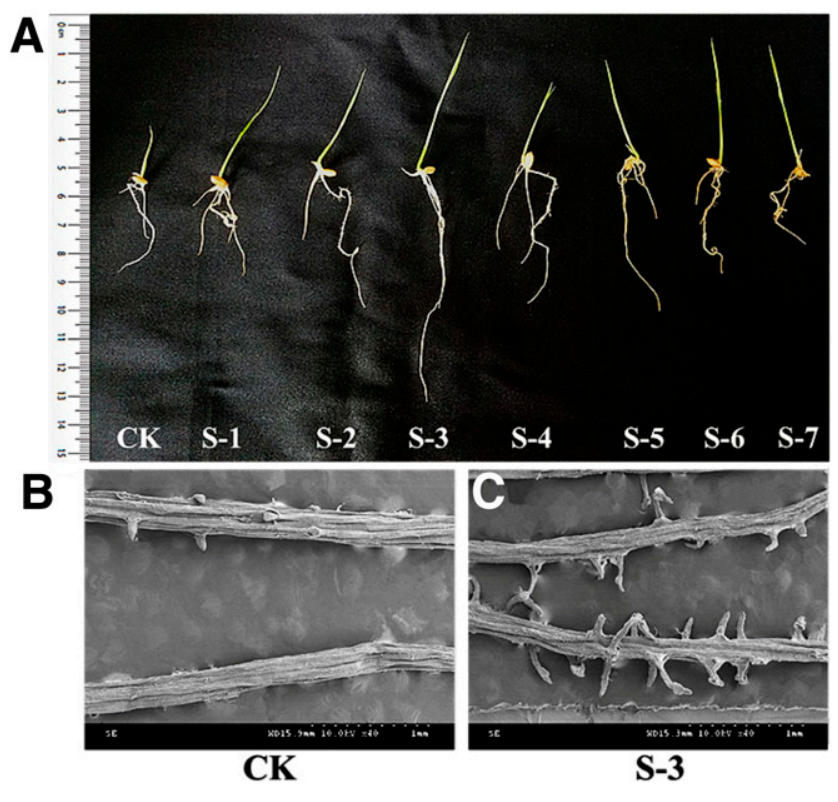

Fig. 1. Effect of strain Bacillus pumilus LZP02 on the growth of rice roots at $48 \mathrm{~h}$ after inoculation with $B$. pumilus LZP02. A, Effects of different strain concentrations on the growth of rice roots (colony-forming units per milliliter), control $(\mathrm{CK})=0, \mathrm{~S}-1=1 \times 10^{7}, \mathrm{~S}-2=2 \times 10^{7}, \mathrm{~S}-3=4 \times 10^{7}, \mathrm{~S}-4=$ $6 \times 10^{7}, \mathrm{~S}-5=8 \times 10^{7}, \mathrm{~S}-6=1 \times 10^{8}$, or S-7 $=1.2 \times 10^{8} . \mathbf{B}$, Effect of CK treatment on rice fibrous roots at $48 \mathrm{~h}$ after inoculation with $B$. pumilus LZP02. C, Effect of S-3 treatment on rice fibrous roots at $48 \mathrm{~h}$ after inoculation with B. pumilus LZP02. 
downregulated) participate in starch and sucrose metabolism, seven (upregulated) participate in fructose and mannose metabolism, amino sugar and nucleotide sugar metabolism, and the pentose phosphate pathway, and two (upregulated) participate in glyoxylate and dicarboxylate metabolism.

To confirm the RNA-seq data, the expressions of some genes,

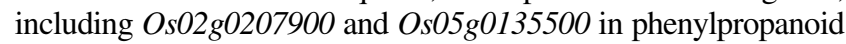
biosynthesis, Os09g0298200, Os01g0174300, and Os08g0526100 in amino sugar and nucleotide sugar metabolism, Os07g0577300, XLOC_000876, and XLOC_029271 in sucrose and starch metabolism, Os08g0495800 and Os03g0115100 in fructose and mannose metabolism, Os03g0102100 and Os $12 g 0291100$ in glyoxylic and dicarboxylic metabolism, and OsO2g0714200 and OsO8gO154300 in the pentose phosphate pathway were measured by reverse transcription-quantitative PCR (RT-qPCR). Consistent with the results of the transcriptomic analysis, the expression levels of genes, including Os09g0298200, Os08g0495800, Os01g0174300, Os03g0115100, Os08g0526100, and Os03g0102100, were changed by B. pumilus LZP02 (Fig. 6C).

\section{Metabolomic differences among CK, S-3, and J (only} B. pumilus LZP02 at $4 \times 10^{7} \mathrm{CFU} / \mathrm{ml}$ without rice).

In order to find out the different metabolites in rice root exudates inoculated with $B$. pumilus LZP02, the metabolomics of $\mathrm{CK}, \mathrm{S}-3$, and $\mathrm{J}$ were analyzed in Murashige and Skoog liquid medium. The significantly altered metabolites $(P<0.05)$ in the CK, S-3, and J treatments were selected for subsequent analysis and were summarized. The principal component analysis (PCA) results for the CK, S-3, and J treatments suggested significant changes in metabolites (Fig. 7), and the differential metabolites between the CK- and S-3-treated plants are illustrated in Figure 8A. The results identified 13 differential metabolites related to phenylpropanoid biosynthesis, starch and sucrose metabolism, the pentose phosphate pathway, and glyoxylate and dicarboxylate metabolism $(P<0.05)$; sucrose, ferulic acid, oleic acid, acetyl-coA, and ribose

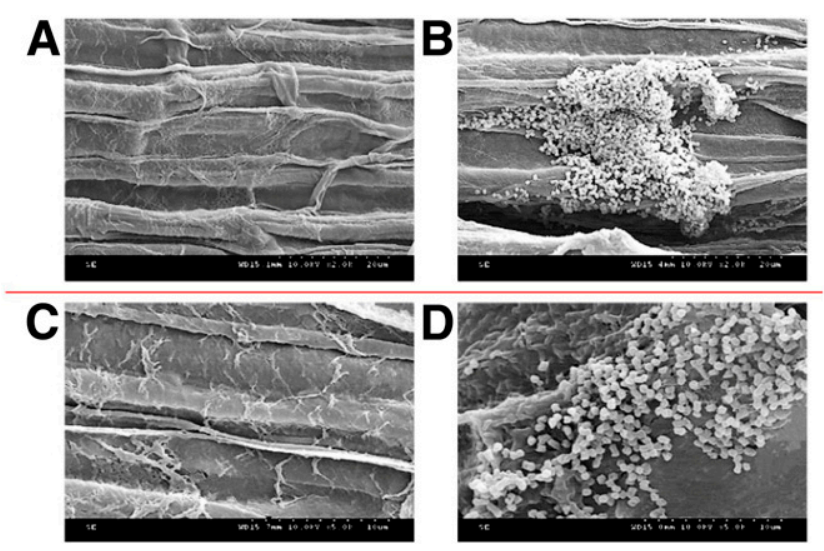

Fig. 3. Scanning electron microscopy images of rice roots at $48 \mathrm{~h}$ after inoculation with Bacillus pumilus LZP02. A, Control (CK) treatment under 2.0-k magnification. B, S-3 treatment under 2.0-k magnification. C, CK treatment under $5.0-\mathrm{k}$ magnification. D, S-3 treatment under $5.0-\mathrm{k}$ magnification.
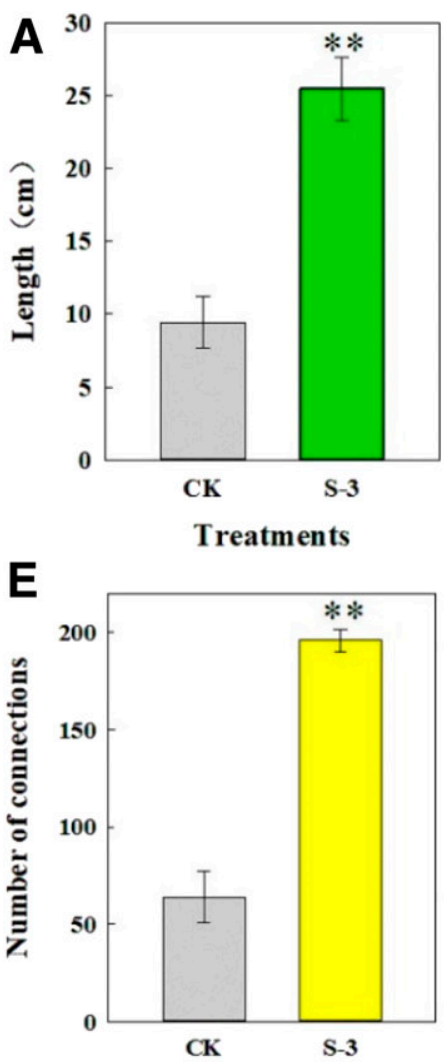

Treatments

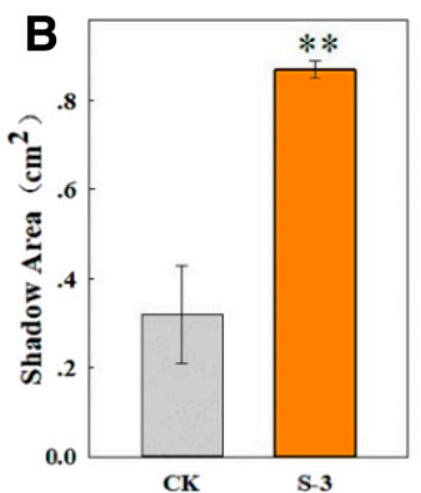

Treatments

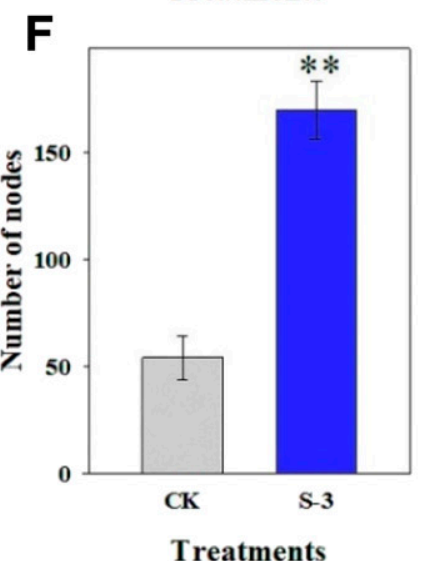

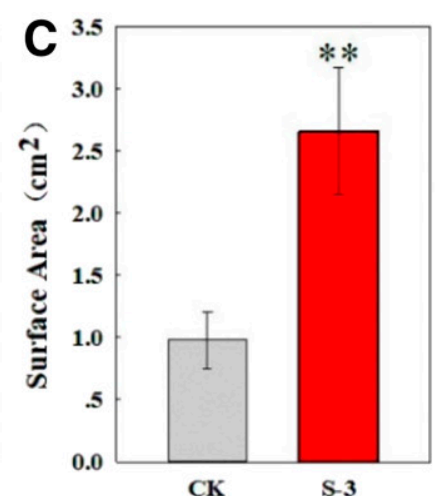

Treatments

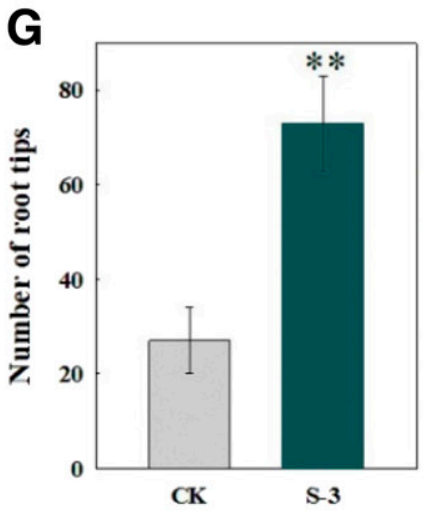

Treatments

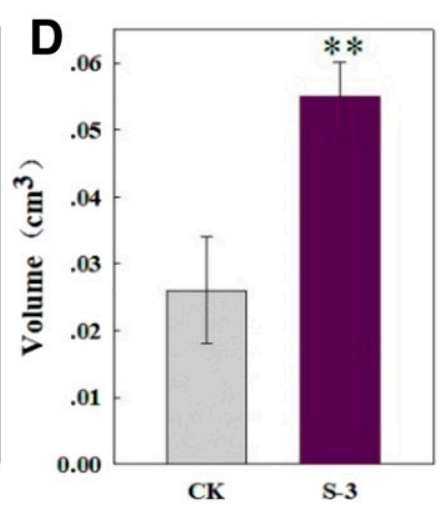

Treatments

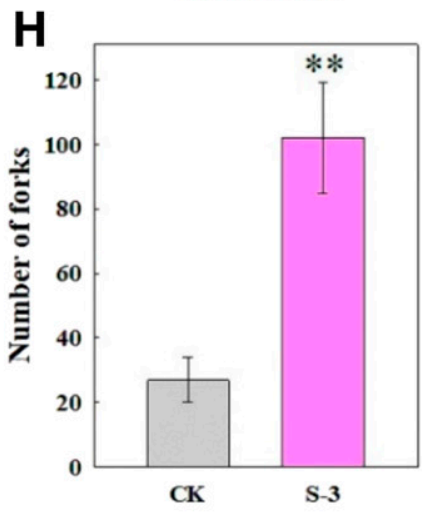

Treatments

Fig. 2. Effect of Bacillus pumilus LZP02 on the growth of rice roots under S-3 treatment at $48 \mathrm{~h}$ after inoculation with B. pumilus LZP02. A, Root length and B, root shadow area (sum of shadow area of the tiled roots per plant). C, Root surface area (total root surface area per plant). D, Root volume (total root volume per plant). E, Number of root connections (total number of overlapping points of different roots of a plant by plane projection). F, Number of root nodes (total number of root nodes per plant). G, Number of root tips (total number of root tips per plant). H, Number of root forks (sum of the number of branching roots per plant). Asterisks $(* *)$ indicate $P<0.01(n=3)$. 
5-phosphate were the most significantly different metabolites between CK and S-3 (Fig. 8B). The differential metabolites between S-3- and $\mathrm{J}$ treatments are shown in Figure $8 \mathrm{C}$. According to the data, nine of the differential metabolites are associated with phenylpropanoid biosynthesis, starch and sucrose metabolism, the pentose phosphate pathway, and glyoxylate and dicarboxylate metabolism $(P<0.05)$, and 3 feruloylquinic acid and feruloyl tyramine were the most significantly different between S-3 and J (Fig. 8D). The malic acid and ferulic acid contents increased in S-3, whereas the malic acid, ferulic acid, and 3-feruloylquinic acid contents increased in $\mathrm{J}$. These findings confirmed the reliability of the metabolomic, transcriptomic, and proteomic analyses at the metabolic, transcriptomic, and protein levels, respectively.

\section{DISCUSSION}

Currently, the demand for rice is gradually increasing, and the growth of rice roots directly affects yield. PGPRs are an important functional group of beneficial bacteria that can be used for plant growth promotion (Lemessa and Zeller 2007). B. pumilus LZP02 promoted the growth of rice roots (Figs. 1 and2). The SEM results clearly indicated the growth status of bacteria and roots and demonstrated that $B$. pumilus LZP02 colonizes rice roots (Fig. 3). B. pumilus LZP02 also improved physiological indicators in rice (Fig. 4), similar to arbuscular mycorrhizal fungi in fava bean (Pereira et al. 2019) and Pedobacter sp. strain CC1 and Bacillus safensis B106 in strawberry (Morais et al. 2019).
Based on the proteomic results (Fig. 5), the DEPs were primarily involved in phenylpropanoid biosynthesis, starch and sucrose metabolism, the pentose phosphate pathway, and glyoxylate and dicarboxylate metabolism. Details regarding the DEPs are provided in Supplementary Table S2. Fifteen DEPs involved in starch and sucrose metabolism were highlighted, including hexokinase, cytosolic starch phosphorylase, cell-wall invertase, $\beta$-glucosidase, vacuolar acid invertase, and fructose6-phosphate 1-phosphotransferase. The expression level of $\beta$-glucosidase, which plays a role in hydrolyzing sucrose to glucose (Pang et al. 2017), was significantly increased, and the level of $0 s 07 \mathrm{~g} 0577300$, encoding a glycoside hydrolase, was also increased in the transcriptome (Fig. 6B and C). The expression of XLOC_029271 was increased in the absence of phosphorus (Zhu et al. 2015). However, the results showed that $B$. pumilus LZP02 downregulated the expression of XLOC_029271 (Fig. 6B and C) and improved phosphorus absorption (Fig. 4A). It could be speculated that the improved phosphorus absorption resulted in the downregulation of XLOC_029271. Sucrose and glucose-6-phosphate, which are important intermediate metabolites in starch and sucrose metabolism, provide a large amount of energy and are closely linked to many other reactions (Ohashi et al. 2018); both exhibited significant changes in the metabolome in the present study (Fig. 8). Therefore, B. pumilus LZP02 could enhance the starch and sucrose metabolism as an intermediate reaction process to provide the energy needed for biological reactions in rice roots (Fig. 9), which was similar to the effect of Pseudomonas putida in maize (SkZ et al. 2018). Through the
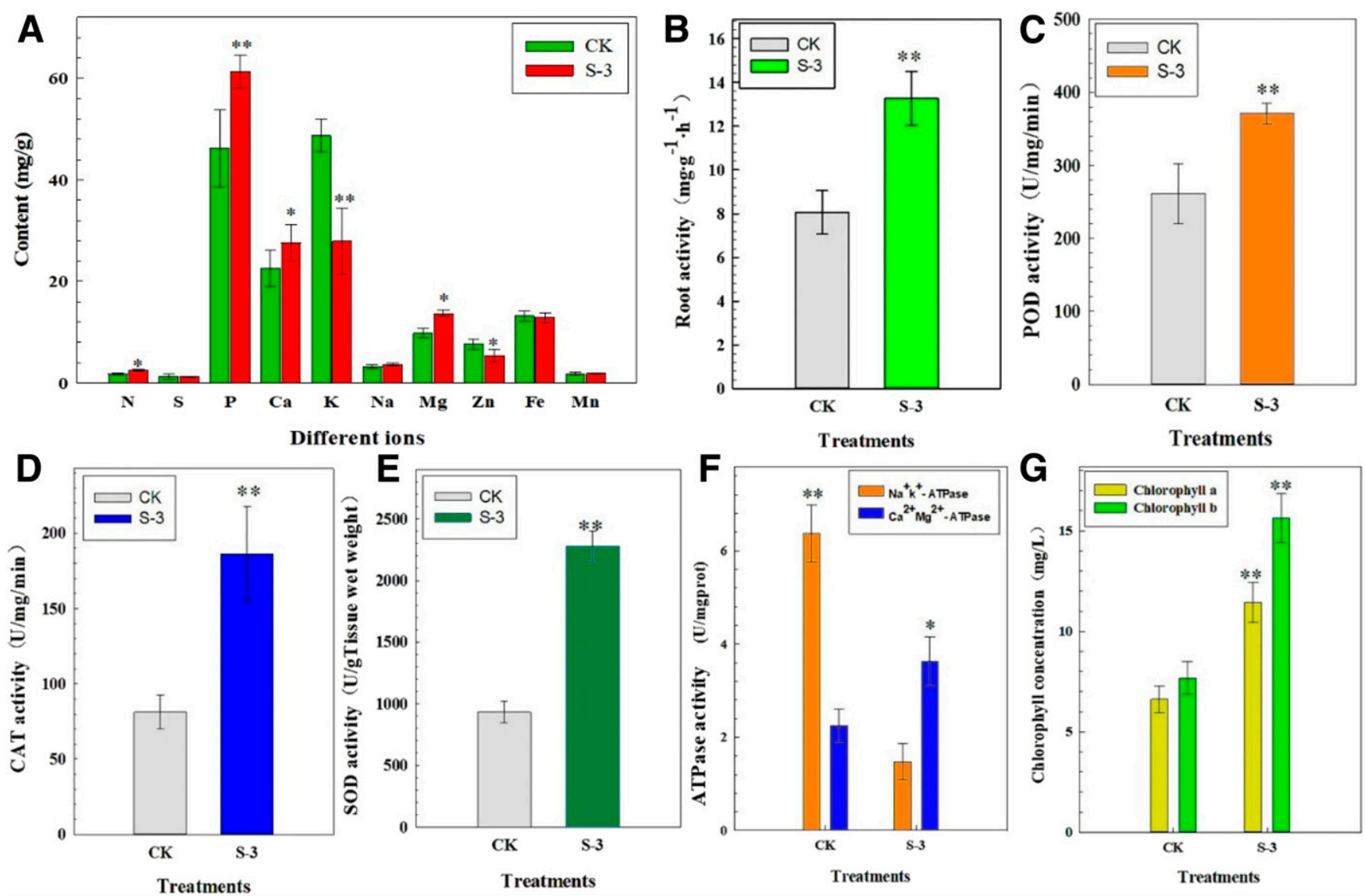

Fig. 4. Effects of Bacillus pumilus LZP02 on the physiological characteristics of rice roots at $48 \mathrm{~h}$ after inoculation with B. pumilus LZP02. A, Effect of S-3 treatment on the absorption of different ions. B, Effect of S-3 treatment on rice root activity; C, peroxidase (POD) activity; D, catalase (CAT) activity; E, superoxide dismutase (SOD) activity; $\mathbf{F}$, adenosine triphosphatase (ATPase) activity; $\mathbf{G}$, chlorophyll concentration. Two asterisks $(* *)$ indicate $P<0.01$ and one (*) indicates $P<0.05(n=3)$. 
proteomic analysis, 20 DEPs were identified in glyoxylate and dicarboxylate metabolism, and the isocitrate lyase level increased significantly. Isocitrate lyase is one of the key enzymes in the unique metabolic process of the glyoxylate cycle, and it catalyzes the decomposition of isocitrate to form glyoxylic acid and succinic acid (Bhusal et al. 2017; Lu et al. 2005). The changes in expression of the Os $12 \mathrm{~g} 0291100$ gene in glyoxylic and dicarboxylic metabolism (of the transcriptome) and the glyoxylic acid content (of the metabolome) were consistent with that of isocitrate lyase (of the proteome). Oleic acid is decomposed by $\beta$-oxidation to form acetyl-CoA (Salam et al. 1989). Glyoxylic acid produces malic acid through acetyl-CoA and dehydrogenation of malic acid produces oxaloacetate, which constitutes the glyoxylate cycle ( $\mathrm{Lu}$ et al. 2005). Important metabolites in pathways, namely, oleic acid, acetylCoA, glyoxylic acid, malic acid, and oxaloacetate, were all significantly upregulated (Fig. 8B). In addition, the malic acid content increased significantly in both $\mathrm{S}-3$ versus $\mathrm{CK}$ and S-3 versus $\mathrm{J}$ (Fig. 8). The increase in metabolites may provide the material required for starch and sucrose metabolism. The proteomic analysis identified 15 DEPs in the pentose phosphate pathway, and ribose 5-phosphate isomerase precursor, an important enzyme in the pentose phosphate pathway, was significantly upregulated (Fig. 5). With regard to the metabolomic results, ribose 5-phosphate was significantly upregulated, as was also confirmed by the proteomic results (Fig. 9). In transcriptomics, the gene Os02g0714200 encoding pyrophosphate-fructose-6-phosphate phosphotransferase was significantly upregulated (Fig. 6B and C). Overexpression of cytosolic invertase results in upregulation of carbohydrates while increasing flux through the pentose phosphate pathway (Centeno et al. 2008). Based on our results, an increase in the flux of the pentose phosphate pathway could provide glucose as an energy source for rice. Overall, upregulation of some metabolic pathways, including starch and sucrose metabolism, the pentose phosphate pathway, and glyoxylate and dicarboxylate metabolism, led to upregulation of carbohydrate metabolism in rice roots (Fig. 9). Therefore, the results indicated that B. pumilus LZP02 could promote the growth of rice roots by enhancing carbohydrate metabolism.

A total of 28 DEPs in phenylpropanoid biosynthesis, including cytosolic aldehyde dehydrogenase, 4-coumarate:coenzyme A ligase, cinnamyl alcohol dehydrogenase, phenylalanine ammonialyase, $\beta$-glucosidase, cytochrome, and POD, were identified (Fig. 5; Supplementary Table S2). 4-Coumarate:coenzyme A ligase is a key enzyme in the phenylpropanoid metabolism pathway in monoalcohols and flavonoid biosynthesis and is related to the synthesis of lignin and ferulic acid in rice (Gui et al. 2011; Sun et al. 2013). Cinnamyl alcohol dehydrogenase is involved in the synthesis of lignin and catalyzes the formation of precursors of lignin monomers from a variety of different cinnamaldehydes (Fan et al. 2009). POD, which is present in peroxisomes, participates in the synthesis of lignin from carbohydrates, thereby protecting cells (Hrubcová et al. 2000). Significant increases were observed in the expression levels of 4-coumarate:coenzyme A ligase, cinnamyl alcohol dehydrogenase, and POD in this study (Supplementary Table S2), suggesting that $B$. pumilus LZP02 could promote lignin synthesis and resilience in rice roots (Fig. 9). At the same time, expression of gene Os05g0135500, encoding POD, was increased in the transcriptome (Fig. 6B and C). Phenylalanine ammonia-lyase (Liu et al. 2018a) and $\beta$-glucosidase (Pengthaisong and Ketudat Cairns 2014) are also important enzymes in the phenylpropanoid biosynthesis pathway. The expression level of phenylpropanoid biosynthesis pathway components correlates positively with the lignin content in Toona sinensis (Sui et al. 2019). Additionally, an increase in the expression of genes that participate in phenylpropanoid biosynthesis increases resistance to fungal pathogens in avocado (Xoca-Orozco et al. 2019). Ferulic acid is an intermediate metabolite that participates in the synthesis of coniferyl alcohol
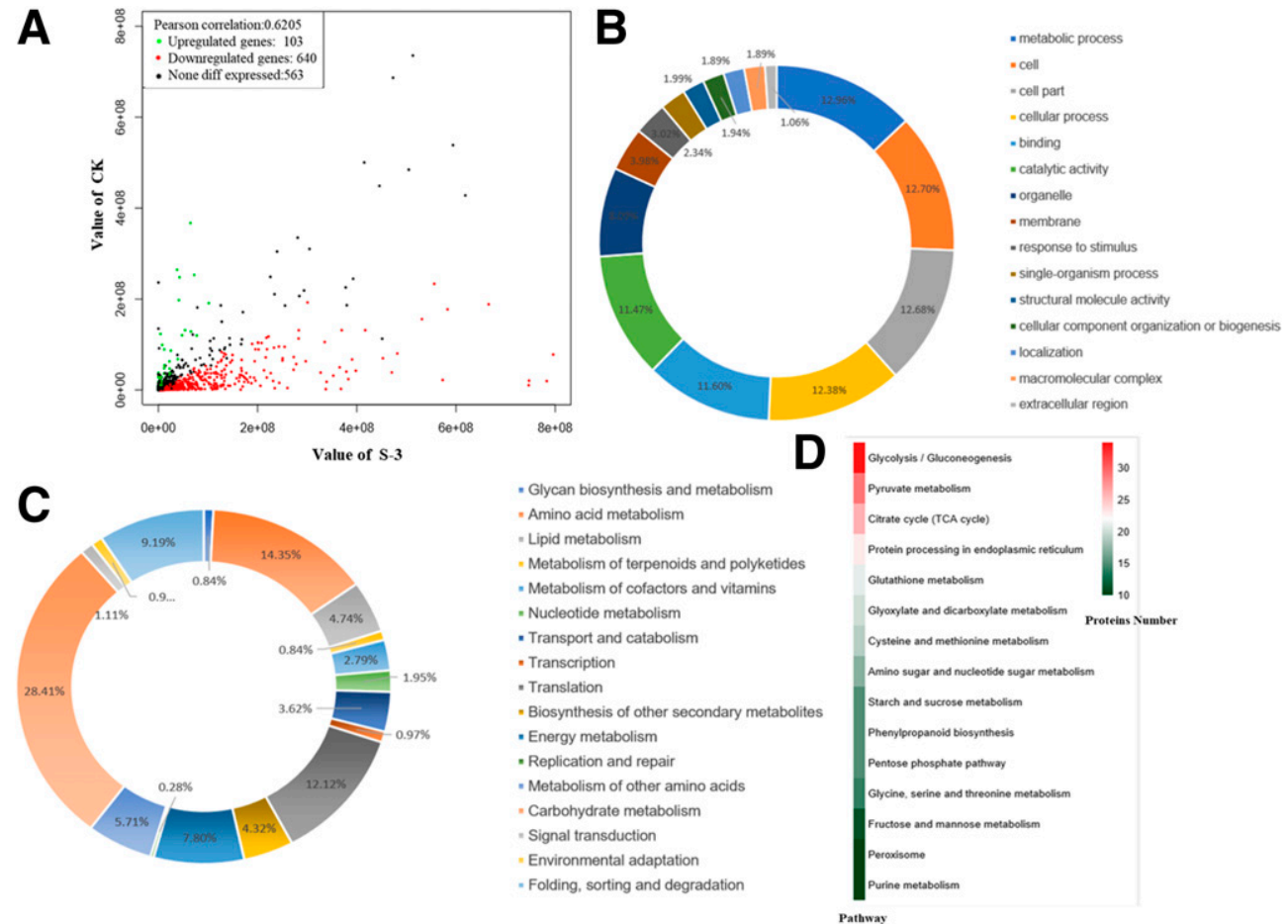

Fig. 5. Effect of Bacillus pumilus LZP02 on the proteome of rice roots at $48 \mathrm{~h}$ after inoculation with B. pumilus LZP02. A, Differential analysis scatter plot of proteins in control (CK) and S-3 treatments; the abscissa and ordinate represent the expression levels of S-3 and CK treatments, respectively. B, Gene ontology analysis of the differentially expressed proteins (DEPs) between CK and S-3. C, KEGG_B analysis of the DEPs between CK and S-3. D, KEGG_C analysis of the DEPs between CK and S-3 (only the first 15 are shown). 
from coniferaldehyde and, ultimately, in the synthesis of lignin and it also exhibits antioxidant activity to protect DNA (Jia et al. 2019). The metabolomic data showed that the addition of B. pumilus LZP02 to rice resulted in a significant increase in ferulic acid; the ferulic acid content was significantly increased in both $\mathrm{S}-3$ versus $\mathrm{CK}$ and S-3 versus $\mathbf{J}$ (Fig. 8). This may be because rice root metabolites and LZP02 metabolites can promote the growth of other organisms (Rasmussen et al. 2020; Toju et al. 2018). Therefore, B. pumilus LZP02 may enzymatically stimulate phenylpropanoid biosynthesis to promote growth in rice roots (Fig. 9).

The study has demonstrated that B. pumilus LZP02 colonizes rice roots and promotes growth in rice roots by improving carbohydrate metabolism and phenylpropanoid biosynthesis (Fig. 9).

\section{MATERIALS AND METHODS}

\section{Materials.}

B. pumilus LZP02 was isolated previously from the rice rhizosphere in northeast China. B. pumilus LZP02 was cultured in beef extract-peptone medium for $24 \mathrm{~h}$, which was collected by centrifuging at $5,600 \times g$ and was stored with $15 \%$ glycerol at $-80^{\circ} \mathrm{C}$. B. pumilus LZP02 was cultured in Luria-Bertani medium for $8 \mathrm{~h}$, and the final cell concentration was $10^{9}$ CFU/ml (Supplementary Fig. S1). The culture was centrifuged, and the cells were suspended in stroke-physiological saline solution $(\mathrm{NaCl} 0.9 \%)$ at $10^{9} \mathrm{CFU} / \mathrm{ml}$ until use.

Rice seeds (Oryza sativa Longgeng 46) were provided by the Rice Research Institute of Heilongjiang Academy of Agricultural Sciences. The seeds were arranged randomly in a growth chamber, with controlled light (cycles of $16 \mathrm{~h}$ of light and $8 \mathrm{~h}$ of dark), temperature $\left(22 \pm 2^{\circ} \mathrm{C}\right)$ and relative humidity (around $70 \%$ ). Sterile water was added twice per week until the seedling stage.

\section{Growth and root analysis.}

Rice seedlings of similar size were selected and were planted in $50 \mathrm{ml}$ of Murashige and Skoog liquid medium. Rice seedlings were treated in the two-leaf one-heart period with $B$. pumilus LZP02. The final concentrations of $B$. pumilus LZP02 in each treatment were as follows: $0 \mathrm{CFU} / \mathrm{ml}(\mathrm{CK})$, $1 \times 10^{7} \mathrm{CFU} / \mathrm{ml}(\mathrm{S}-1), 2 \times 10^{7} \mathrm{CFU} / \mathrm{ml}(\mathrm{S}-2), 4 \times 10^{7} \mathrm{CFU} / \mathrm{ml}$ $(\mathrm{S}-3), 6 \times 10^{7} \mathrm{CFU} / \mathrm{ml}(\mathrm{S}-4), 8 \times 10^{7} \mathrm{CFU} / \mathrm{ml}(\mathrm{S}-5), 1 \times 10^{8}$ $\mathrm{CFU} / \mathrm{ml}(\mathrm{S}-6)$, or $1.2 \times 10^{8} \mathrm{CFU} / \mathrm{ml}$ (S-7). All the operations were performed under aseptic conditions.

To determine the $B$. pumilus LZP02 concentration most suitable for rice seedling root growth, data on the roots, including root length, root shadow area, root surface area, root

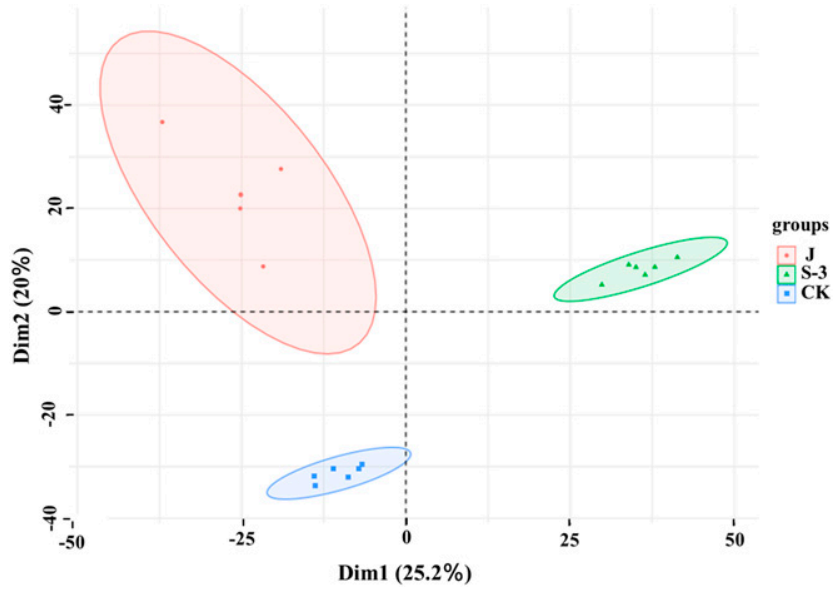

Fig. 7. A principal component analysis score plot for the metabolomics data in control (CK), S-3, and $\mathrm{J}$ treatments at $48 \mathrm{~h}$ after inoculation with Bacillus pumilus LZP02 through a metabolomic analysis.

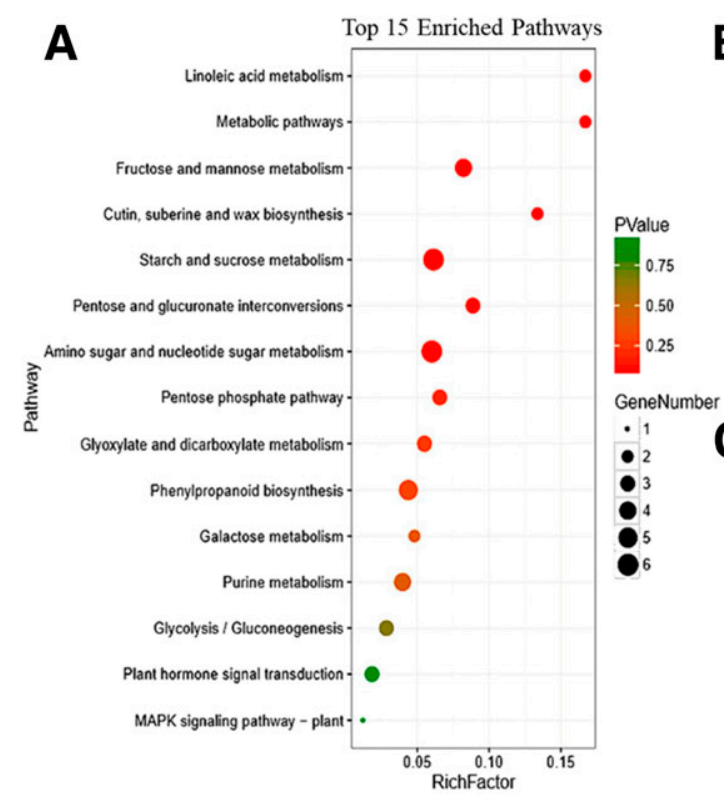

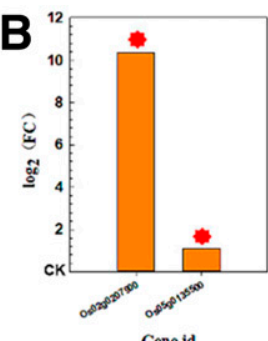
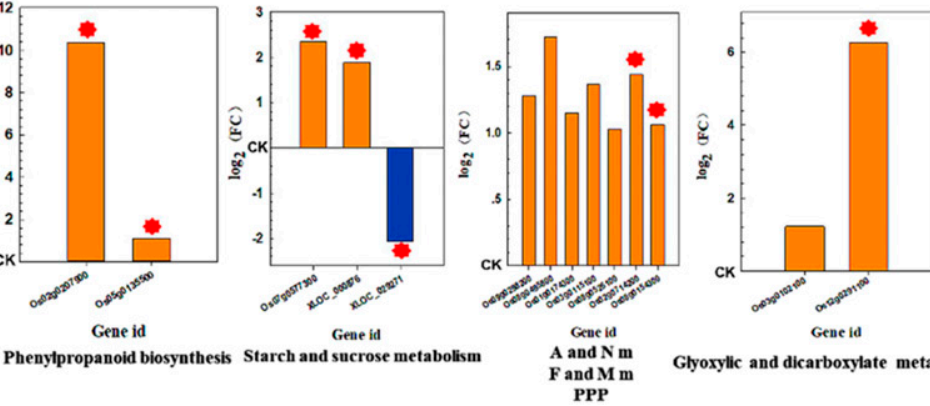

C
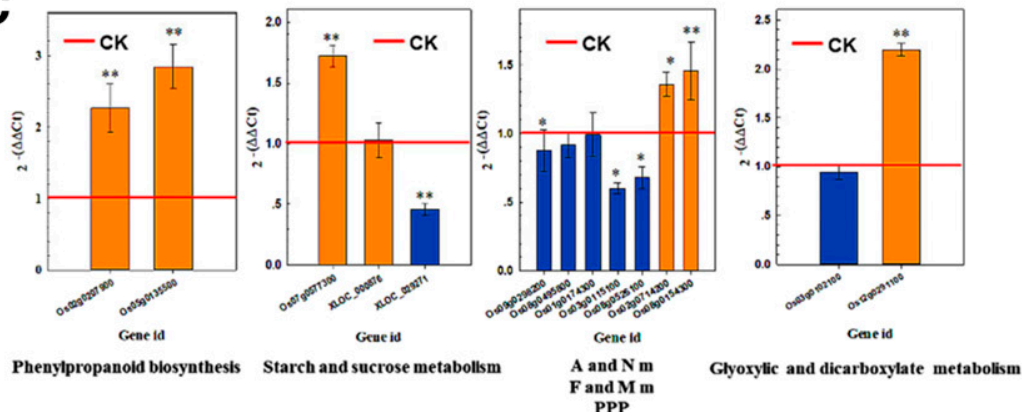

Fig. 6. Effect of Bacillus pumilus LZP02 on the transcriptome of rice roots at $48 \mathrm{~h}$ after inoculation with B. pumilus LZP02. A, KEGG_C analysis of differentially expressed genes in the control $(\mathrm{CK})$ and $\mathrm{S}-3 . \mathbf{B}, \mathrm{Log}_{2}$ fold change values of differentially expressed genes in the transcriptome. C, Gene expression of differentially expressed genes by reverse transcription-quantitative (RT-q)PCR. Two asterisks (**) indicate $P<0.01$ and one $(*)$ indicates $P<$ $0.05(n=3)$; red dots indicate that the transcriptome is identical to the RT-qPCR result. A and $\mathrm{N}$ m $=$ amino sugar and nucleotide sugar metabolism; F and M m $=$ fructose and mannose metabolism; PPP = pentose phosphate pathway. In B and C, yellow represents upregulated genes, and blue represents downregulated genes. 
volume, number of root connections, number of root nodes, number of root tips, and number of root forks, were observed and were measured, at $48 \mathrm{~h}$ after inoculation, using a Tuopu root analyzer (RootAnalysis, PM-Tech).

\section{SEM.}

SEM was performed as previously described (Rashid et al. 2018). Rice roots in the two-leaf one-heart period were randomly collected from the CK and S-3 treatments at $48 \mathrm{~h}$ after inoculation with $B$. pumilus LZP02; 2- to 3-mm-thick samples were adhered to a copper sample table by using a conductive adhesive. A HUS-5GB-type vacuum coating apparatus was used to plate the sections, and the samples were subjected to SEM (S-3400, Hitachi) to observe different parts of the rice root system and obtain images.

\section{Physiological index determination.}

The POD, SOD, CAT, and ATPase activities in rice roots were measured using corresponding test kits (Nanjing Jiancheng Biological Engineering Institute). The ion absorption content was determined using an ion absorption meter (Yoshihara et al. 2019). The chlorophyll content was determined according to the method described by Sakaigaichi et al. (2019). Root activity was determined according to the method described by Whalley et al. (2017).

\section{Proteomic assay.}

Protein extraction of rice roots inoculated with CK and S-3 treatments cultured for $48 \mathrm{~h}$ was performed using the cold acetone method. Roots ( $2 \mathrm{~g}$ ) were cut into approximately $5 \mathrm{~cm}$ pieces, were ground to a powder in liquid nitrogen, and were then dissolved in $2 \mathrm{ml}$ of lysis buffer ( $8 \mathrm{M}$ urea, $2 \%$ sodium dodecyl sulfate [SDS], $1 \times$ protease inhibitor cocktail [Roche Ltd.]), followed by sonication on ice for $30 \mathrm{~min}$ and centrifugation at $9,520 \times g$ for $30 \mathrm{~min}$ at $4^{\circ} \mathrm{C}$. Each treatment was repeated three times. The supernatant was transferred to a fresh tube. For each sample, proteins were precipitated overnight with ice-cold acetone at $-20^{\circ} \mathrm{C}$. The precipitates were washed with acetone three times and were redissolved in $8 \mathrm{M}$ urea, followed by sonication on ice. Protein quality was examined by SDS-polyacrylamide gel electrophoresis.

A BCA protein assay kit (Pierce) was used to determine the protein concentration of the supernatant. A $100-\mu \mathrm{g}$ sample of protein per condition was transferred into a new tube, and the volume was adjusted to $100 \mu \mathrm{l}$ with $8 \mathrm{M}$ urea; $11 \mu \mathrm{l}$ of $1 \mathrm{M} \mathrm{DL}-$ dithiothreitol was added, and the samples were incubated at $37^{\circ} \mathrm{C}$ for $1 \mathrm{~h}$ and were transferred into a 10-K ultrafiltration tube (Merck Millipore). To remove the urea, the samples were washed three times, using $100 \mathrm{mM}$ triethylammonium bicarbonate with centrifugation. A total of $120 \mu \mathrm{l}$ of $55 \mathrm{mM}$ iodoacetamide was added to the samples, which were then incubated at room temperature for $20 \mathrm{~min}$, protected from light. The proteins were digested overnight with sequence-grade modified trypsin (Promega).

Each sample was divided into three equal parts and was resuspended in $30 \mu \mathrm{l}$ of solvent $\mathrm{C}(\mathrm{C}$ : water with $0.1 \%$ formic acid, D: ACN with $0.1 \%$ formic acid), was separated by nanoLC (liquid chromatography), and was analyzed by online electrospray tandem mass spectrometry (MS/MS). The
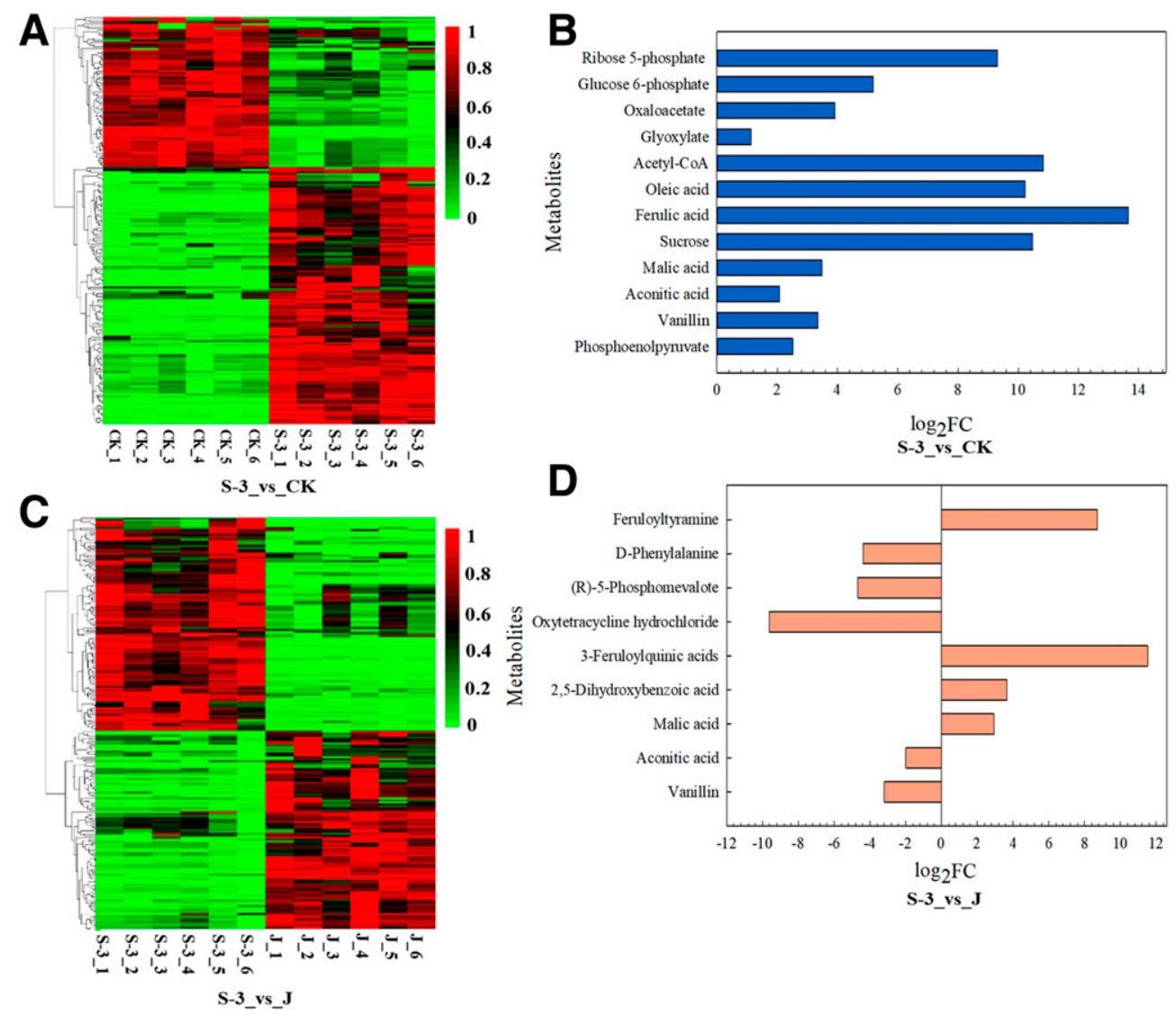

Fig. 8. Analysis of differential and specific metabolites of different treatments at $48 \mathrm{~h}$ after inoculation with Bacillus pumilus LZP02. A, Heat map analysis of S-3 versus control (CK) differential metabolites. B, Significantly different metabolites in phenylpropanoid biosynthesis, starch and sucrose metabolism, the pentose phosphate pathway, and glyoxylate and dicarboxylate metabolism between CK and S-3. C, Heat map analysis of S-3 versus J differential metabolites. D, Significantly different metabolites in phenylpropanoid biosynthesis, starch and sucrose metabolism, the pentose phosphate pathway, and glyoxylate and dicarboxylate metabolism between S-3 and J. 
experiments were performed using an Easy-nLC 1000 system (Thermo Fisher Scientific) connected to a Q Exactive mass spectrometer (Thermo Fisher Scientific) equipped with an online nanoelectrospray ion source. A $10-\mu \mathrm{l}$ peptide sample was loaded onto the trap column (Thermo Scientific Acclaim PepMap C18, $100 \mu \mathrm{m} \times 2 \mathrm{~cm}$ ) with a flow rate of $10 \mu \mathrm{l} / \mathrm{min}$ for 3 min and was subsequently separated on an analytical column (Acclaim PepMap C18, $75 \mu \mathrm{m} \times 15 \mathrm{~cm}$ ) with a linear gradient from $3 \% \mathrm{D}$ to $32 \% \mathrm{D}$ in $120 \mathrm{~min}$. The column was reequilibrated under the initial conditions for $10 \mathrm{~min}$. The column flow rate was maintained at $300 \mathrm{nl} / \mathrm{min}$. An electrospray voltage of $2 \mathrm{kV}$ against the inlet of the mass spectrometer was used. The mass spectrometer was operated in the data-dependent acquisition mode to switch automatically between MS and MS/MS acquisition. Full-scan MS spectra $(\mathrm{m} / \mathrm{z}, 350$ to 1,550$)$ were acquired with a mass resolution of $35 \mathrm{~K}$, followed by sequential high-energy collisional dissociation MS/MS scans with a resolution of 17.5. The dynamic exclusion time was set as $20 \mathrm{~s}$.

MS/MS spectra were extracted, charge-state deconvoluted, and deisotoped. The MS data were then transformed into MGF files with Proteome Discovery 1.2 (Thermo) and processed by MaxQuant v1.5. PEAKS DB was set up for a search using trypsin as the digestion enzyme with a parent ion tolerance of $7.0 \mathrm{ppm}$. Carbamidomethylation was specified as a fixed modification. Oxidation (M) and acetylation (protein N-term) were specified as variable modifications. Peptides were filtered with a $1 \%$ false discovery rate (FDR).

Protein identifications were accepted if they contained at least two identified peptides. Proteins that contained similar peptides and could not be differentiated based on MS/MS analysis alone were grouped to satisfy the principles of parsimony. iBAQ was employed for peptide and protein abundance calculations. Proteins with a fold change in a comparison $>1.2$ or $<0.83$ and unadjusted significance level $P<0.05$ were considered to be differentially expressed. The proteins were annotated against the GO and KEGG databases to identify functions. Significant GO and KEGG functions and pathways were examined for DEPs with $P \leq 0.05$.

\section{RNA-seq.}

For this experiment, roots ( $2 \mathrm{~g}$ each treatment) from $\mathrm{CK}$ and S-3 cultured for $48 \mathrm{~h}$ after incubation with B. pumilus LZP02 were sent to Gene Denovo Biotechnology Co. for RNA extraction, library construction, RNA-seq, and primary data analysis. Each treatment was repeated three times. In brief, RNA was extracted from the rice root system, using a TRIzol kit according to manufacturer instructions (Promega), and was mixed in equal quantities for sequencing. After total RNA was extracted, eukaryotic messenger RNA (mRNA) was enriched with oligo (dT) beads, and prokaryotic mRNA was enriched by removing ribosomal RNA (rRNA), using the Ribo-Zero magnetic kit (Epicentre). The enriched mRNA was fragmented into short fragments using fragmentation buffer and was reverse transcribed into cDNA with random primers. Second-strand cDNA was synthesized by using DNA polymerase I, RNase H, dNTP, and buffer. The cDNA fragments were purified with the QiaQuick PCR extraction kit, were end-repaired and poly(A)treated, and were ligated to Illumina sequencing adapters. The ligation products were size-selected by agarose gel electrophoresis, were PCR-amplified, and were sequenced using Illumina HiSeqTM 2500 (Gene De Novo Biotechnology Co.).

Reads obtained from sequencing include raw reads containing adapters or low-quality bases, which affect the subsequent assembly and analysis. Thus, to obtain high-quality clean reads, the reads were further filtered as follows: i) reads containing adapters were removed, ii) reads containing more than $10 \%$ unknown nucleotides $(\mathrm{N})$ were removed, and iii) low-quality reads containing more than $50 \%$ low-quality $(Q$ value $\leq 20)$ bases were removed. The short-read alignment tool Bowtie2 (Langmead and Salzberg 2012) was used for mapping reads to the rRNA database, and the rRNA-mapped reads were removed. The remaining reads were further used for assembly and analysis of the transcriptome. The rRNA-removed reads of each sample were mapped to the reference genome by TopHat2 (version 2.0.3.12) (Kim et al. 2013). After alignment with the reference genome, unmapped reads (or those mapped very poorly) were realigned with Bowtie2, and the enriched unmapped reads were split into smaller segments, which were then used to find potential splice sites. The section and the section position of these short segments were predicted as well. A set of splice sites was built with the initial unmapped reads by TopHat2, without relying on known gene annotations (Trapnell et al. 2010). Transcript reconstruction was carried out with Cufflinks software (Trapnell et al. 2012).

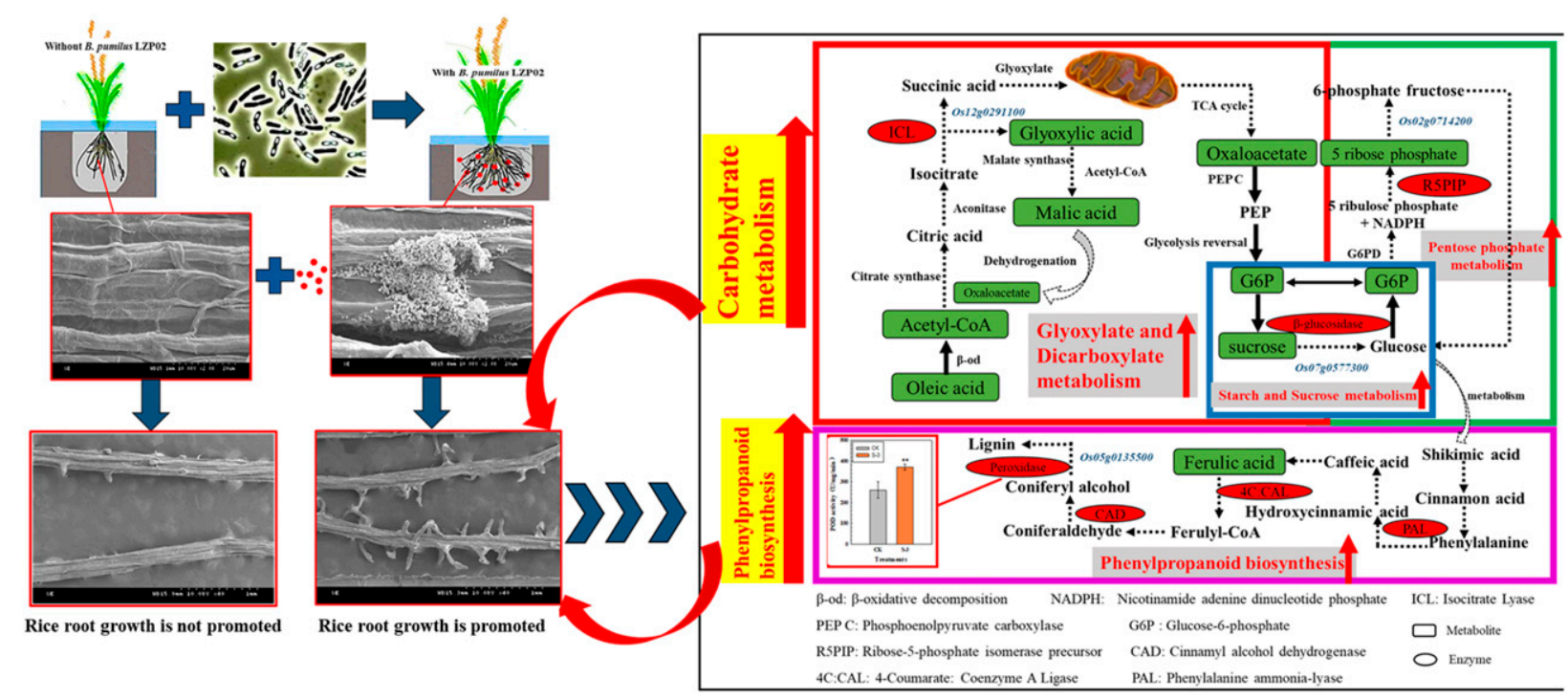

Fig. 9. Schematic diagram of Bacillus pumilus LZP02-mediated promotion of the growth of rice roots, which was summarized through the genes and proteins that were significantly expressed upon treatment. The solid black arrows indicate that metabolites at both ends were detected, and the dashed arrows indicate that metabolites at both ends were not detected or only metabolites at one end of the arrow were detected. 
The edgeR package (available online) was used to identify DEGs across samples or groups. We considered genes with fold change $\geq 2$ and FDR $<0.05$ in a comparison as significant DEGs. The DEGs were then subjected to enrichment analysis of functions and KEGG pathways.

\section{RT-qPCR analysis.}

Rice roots from CK and S-3 cultured for $48 \mathrm{~h}$ after incubation with $B$. pumilus LZP02 were collected, and total cellular RNA was extracted using a UNlQ-10 Column TRIzol total RNA isolation kit (Sangon Biotech) according to the manufacturer protocol. Each treatment was repeated three times. The purified RNA was reverse transcribed into complementary DNA (cDNA) by RevertAid Premium RT (Thermo Scientific), and the cDNA samples were stored at $-70^{\circ} \mathrm{C}$ until use. Each RTqPCR mixture $(20 \mu \mathrm{l})$ contained $10 \mu \mathrm{l}$ of $2 \times$ SG Fast qPCR master mix (High Rox) (Taq DNA polymerase, SYBR green I dye, $\mathrm{MgCl}_{2}$, dNTP mix), $0.4 \mu \mathrm{l}$ of forward primer $(10 \mu \mathrm{M})$, $0.4 \mu \mathrm{l}$ of reverse primer $(10 \mu \mathrm{M}), 2 \mu \mathrm{l}$ of cDNA template (diluted 10 times), and $7.2 \mu \mathrm{l}$ of double distilled $\mathrm{H}_{2} \mathrm{O}$. RT-qPCR was carried out using a StepOne Plus real-time PCR instrument (ABI). Gene expression was determined from three replicates of a single real-time PCR experiment. The primers used for RTqPCR are shown in Supplementary Table S3. The expression ratio was recorded as the fold difference in the quantity of realtime PCR products from $\mathrm{CK}$ and $\mathrm{S}-3$. The relative abundance for each assayed mRNA versus a constitutively expressed gene (16S rRNA) was determined according to the $2^{-\Delta \Delta \mathrm{Ct}}$ method (Z. Wang et al. 2019a and b).

\section{Metabolomic assay in medium.}

The metabolomics of $\mathrm{CK}, \mathrm{S}-3$, and $\mathrm{J}$ were analyzed in Murashige and Skoog liquid medium. For this assay, 50-ml samples of CK, S-3, and J treatments were collected in 50-ml EP tubes. After the addition of $1,000 \mu \mathrm{l}$ of extract solvent (acetonitrile-methanol-water, 2:2:1, containing internal standard), the samples were vortexed for $30 \mathrm{~s}$, were homogenized at $45 \mathrm{~Hz}$ for $4 \mathrm{~min}$, and were sonicated for $5 \mathrm{~min}$ in an ice-water bath. The homogenization and sonication cycle was repeated three times, followed by incubation at $-20^{\circ} \mathrm{C}$ for $1 \mathrm{~h}$ and centrifugation at $8,044 \times g$ and $4^{\circ} \mathrm{C}$ for $15 \mathrm{~min}$. The resulting supernatants were transferred to LC-MS vials and stored at $-80^{\circ} \mathrm{C}$ until ultra-high performance liquid chromatography (UHPLC)-QE Orbitrap/MS analysis. The quality control (QC) sample was prepared by mixing equal aliquots of the supernatants from all the samples. LC-MS/MS analyses were performed using a 1290 UHPLC system (Agilent Technologies) with a UPLC HSS T3 column $(2.1 \times$ $100 \mathrm{~mm}, 1.8 \mu \mathrm{m}$ ) coupled to a Q Exactive instrument (Orbitrap MS, Thermo). Mobile phase A was $0.1 \%$ formic acid in water for positive ion mode and $5 \mathrm{mmol}$ of ammonium acetate per liter in water for negative ion mode; mobile phase $\mathrm{B}$ was acetonitrile. The elution gradient was set as follows: $0 \mathrm{~min}, 1 \% \mathrm{~B} ; 1 \mathrm{~min}, 1 \% \mathrm{~B}$; $8 \mathrm{~min}, 99 \% \mathrm{~B} ; 10 \mathrm{~min}, 99 \% \mathrm{~B} ; 10.1 \mathrm{~min}, 1 \% \mathrm{~B}$; and $12 \mathrm{~min}, 1 \%$ $\mathrm{B}$. The flow rate was $0.5 \mathrm{ml} / \mathrm{min}$. The injection volume was $2 \mu \mathrm{l}$. The QE mass spectrometer was used due to its ability to acquire MS/MS spectra on an information-dependent basis during an LC/MS experiment. In this mode, the acquisition software (Xcalibur 4.0.27, Thermo) continuously evaluates the full-scan survey MS data as it collects and triggers the acquisition of MS/MS spectra depending on preselected criteria. Electrospray ionization source conditions were set as follows: sheath gas flow rate, 45 Arb; aux gas flow rate, 15 Arb; capillary temperature, $320^{\circ} \mathrm{C}$; full MS resolution, 70,000; MS/MS resolution, 17,500; collision energy, 20/40/60 eV in the NCE model; spray voltage, $3.8 \mathrm{kV}$ (positive) or $-3.1 \mathrm{kV}$ (negative).

MS raw data (.raw) files were converted to the mzML format using Proteo Wizard and were processed by R package XCMS (version 3.2), including retention time alignment, peak detection, and peak matching. The data were filtered based on the following criterion: the number of samples containing a metabolite was less than $50 \%$ of the total number of samples in a group (QC samples were also taken as a group). Normalization to an internal standard (Roberts et al. 2012) was subsequently performed for each sample. Next, missing values were replaced by half of the minimum value found in the dataset by default (Xia et al. 2009). The preprocessing results were used to generate a data matrix that consisted of the retention time, mass-to-charge ratio $(\mathrm{m} / \mathrm{z})$, and peak intensity. OSI-SMMS (version 1.0) (Dalian Chem Data Solution Information Technology Co., Ltd.) was applied for peak annotation after data processing with an in-house MS/MS database.

For preliminary visualization of differences between different groups of samples, the unsupervised dimensionality reduction method PCA was applied to all samples using R package models. PCA is a statistical procedure that converts hundreds of thousands of correlated metabolite variables into a set of linearly uncorrelated variables called principal components.

A variable importance in the projection (VIP) score of the $(\mathrm{O})$ PLS model was applied to rank the metabolites that best distinguished the two groups. The VIP threshold was set to 1 . In addition, the $t$ test was used in univariate analysis for screening differential metabolites. Metabolites with a $P$ value of $t$ test $<0.05$ and $V I P \geq 1$ were considered differential metabolites between the two groups.

Metabolites were mapped to KEGG metabolic pathways for pathway enrichment analysis, identifying significantly enriched metabolic pathways or signal transduction pathways associated with the differential metabolites compared with the entire background. The calculation formula is as follows:

$$
P=1-\sum_{i=0}^{m-1} \frac{\left(\begin{array}{c}
m \\
i
\end{array}\right)\left(\begin{array}{c}
N-M \\
n-i
\end{array}\right)}{\left(\begin{array}{c}
N \\
n
\end{array}\right)}
$$

where $N$ is the total number of metabolites with KEGG annotations, $n$ is the number of differential metabolites in $N, M$ is the total number of metabolites annotated to specific pathways, and $m$ is the number of differential metabolites in $M$. The calculated $P$ value was subjected to FDR correction, with FDR $\leq 0.05$ as a threshold. Pathways meeting this condition were defined as significantly enriched pathways associated with the differential metabolites.

\section{Statistical analyses.}

All statistical analyses were performed using SPSS 22.0 (IBM). The differences in the means were determined using Turkey's test, with $P<0.05$ or $P<0.01(n=3)$ denoting statistical significance.

\section{AUTHOR-RECOMMENDED INTERNET RESOURCES}

edgeR package: https://www.rdocumentation.org/packages/edgeR R package models: http://www.r-project.org

\section{LITERATURE CITED}

Abdallah, Y., Yang, M., Zhang, M., Masum, M. M. I., Ogunyemi, S. O., Hossain, A., An, Q., Yan, C., and Li, B. 2019. Plant growth promotion and suppression of bacterial leaf blight in rice by Paenibacillus polymyxa Sx3. Lett. Appl. Microbiol. 68:423-429.

Arriel-Elias, M. T., de Carvalho Barros Côrtes, M. V., de Sousa, T. P., Chaibub, A. A., and de Filippi, M. C. C. 2019. Induction of resistance in rice plants using bioproducts produced from Burkholderia pyrrocinia BRM 32113. Environ. Sci. Pollut. Res. Int. 26:19705-19718.

Bhusal, R. P., Patel, K., Kwai, B. X. C., Swartjes, A., Bashiri, G., Reynisson, J., Sperry, J., and Leung, I. K. H. 2017. Development of 
NMR and thermal shift assays for the evaluation of Mycobacterium tuberculosis isocitrate lyase inhibitors. MedChemComm 8:2155-2163.

Centeno, D. C., Oliver, S. N., Nunes-Nesi, A., Geigenberger, P., Machado, D. N., Loureiro, M. E., Silva, M. A., and Fernie, A. R. 2008. Metabolic regulation of pathways of carbohydrate oxidation in potato (Solanum tuberosum) tubers. Physiol. Plant. 133:744-754.

Chaudhari, P. R., Tamrakar, N., Singh, L., Tandon, A., and Sharma, D. 2018. Rice nutritional and medicinal properties: A review article. J. Pharmacogn. Phytochem. 7:150-156.

Fan, L., Shi, W. J., Hu, W. R., Hao, X. Y., Wang, D. M., Yuan, H., and Yan, H. Y. 2009. Molecular and biochemical evidence for phenylpropanoid synthesis and presence of wall-linked phenolics in cotton fibers. J. Integr. Plant Biol. 51:626-637.

Gui, J., Shen, J., and Li, L. 2011. Functional characterization of evolutionarily divergent 4-coumarate:coenzyme a ligases in rice. Plant Physiol. 157:574-586.

Hermosa, R., Viterbo, A., Chet, I., and Monte, E. 2012. Plant-beneficial effects of Trichoderma and of its genes. Microbiology 158:17-25.

Hrubcová, M., Cvikrová, M., Eder, J., Zoń, J., and Macháčková, I. 2000. Effect of inhibition of phenylpropanoid biosynthesison peroxidase and IAA-oxidase activities and auxin contentin alfalfa suspension cultures. Plant Physiol. Biochem. 38:949-956.

Jia, X., Zhao, M., Xia, N., Teng, J., Jia, C., Wei, B., Huang, L., and Chen, D. 2019. Interaction between plant phenolics and rice protein improved oxidative stabilities of emulsion. J. Cereal Sci. 89:102818.

Khan, A., Zhao, X. Q., Javed, M. T., Khan, K. S., Bano, A., Shen, R. F., and Masood, S. 2016. Bacillus pumilus enhances tolerance in rice (Oryza sativa $\mathrm{L}$.) to combined stresses of $\mathrm{NaCl}$ and high boron due to limited uptake of $\mathrm{Na}^{+}$. Environ. Exp. Bot. 124:120-129.

Kim, D., Pertea, G., Trapnell, C., Pimentel, H., Kelley, R., and Salzberg, S. L. 2013. TopHat2: Accurate alignment of transcriptomes in the presence of insertions, deletions and gene fusions. Genome Biol. 14:R36.

Langmead, B., and Salzberg, S. L. 2012. Fast gapped-read alignment with Bowtie 2. Nat. Methods 9:357-359.

Lemessa, F., and Zeller, W. 2007. Screening rhizobacteria for biological control of Ralstonia solanacearum in Ethiopia. Biol. Control 42:336-344.

Li, H., Qiu, Y., Yao, T., Ma, Y., Zhang, H., and Yang, X. 2020. Effects of PGPR microbial inoculants on the growth and soil properties of Avena sativa, Medicago sativa, and Cucumis sativus seedlings. Soil Tillage Res. 199:104577.

Liu, X., He, Z., Yin, Y., Xu, X., Wu, W., and Li, L. 2018a. Transcriptome sequencing and analysis during seed growth and development in Euryale ferox Salisb. BMC Genomics 19:343.

Liu, Z. P., Wang, Z. G., Xu, W. H., Chen, W. J., Lv, Z. H., Long, W. C., and Shi, Y. R. 2018b. Screen, identification and analysis on the growthpromoting ability for the rice growth-promoting rhizobacteria. J. Agric. Resour. Environ. 35:119-125.

Lu, Y., Wu, Y. R., and Han, B. 2005. Anaerobic induction of isocitrate lyase and malate synthase in submerged rice seedlings indicates the important metabolic role of the glyoxylate cycle. Acta Biochim. Biophys. Sin. (Shanghai) 37:406-414.

Morais, M. C., Mucha, Â., Ferreira, H., Gonçalves, B., Bacelar, E., and Marques, G. 2019. Comparative study of plant growth-promoting bacteria on the physiology, growth and fruit quality of strawberry. J. Sci. Food Agric. 99:5341-5349.

Naik, K., Mishra, S., Srichandan, H., Singh, P. K., and Sarangi, P. K. 2019 Plant growth promoting microbes: Potential link to sustainable agriculture and environment. Biocatal. Agric. Biotechnol. 21:101326.

Nayak, M., Swain, D. K., and Sen, R. 2019. Strategic valorization of de-oiled microalgal biomass waste as biofertilizer for sustainable and improved agriculture of rice (Oryza sativa L.) crop. Sci. Total Environ. 682:475-484.

Ohashi, M., Ishiyama, K., Kusano, M., Fukushima, A., Kojima, S., Hayakawa, T., and Yamaya, T. 2018. Reduction in sucrose contents by downregulation of fructose-1,6-bisphosphatase 2 causes tiller outgrowth cessation in rice mutants lacking glutamine synthetase1;2. Rice (N. Y.) 11:65.

Pang, P., Cao, L. C., Liu, Y. H., Xie, W., and Wang, Z. 2017. Structures of a glucose-tolerant $\beta$-glucosidase provide insights into its mechanism. J. Struct. Biol. 198:154-162.

Pengthaisong, S., and Ketudat Cairns, J. R. 2014. Effects of active site cleft residues on oligosaccharide binding, hydrolysis, and glycosynthase activities of rice BGlu1 and its mutants. Protein Sci. 23:1738-1752.

Pereira, S., Mucha, Â., Gonçalves, B., Bacelar, E., Látr, A., Ferreira, H., Oliveira, I., Rosa, E., and Marques, G. 2019. Improvement of some growth and yield parameters of faba bean (Vicia faba) by inoculation with Rhizobium laguerreae and arbuscular mycorrhizal fungi. Crop Pasture Sci. 70:595-605.

Rashid, N., Zafar, M., Ahmad, M., Malik, K., Haq, I. U., Shah, S. N., Mateen, A., and Ahmed, T. 2018. Intraspecific variation in seed morphology of tribe vicieae (Papilionoidae) using scanning electron microscopy techniques. Microsc. Res. Tech. 81:298-307.

Rasmussen, P. U., Bennett, A. E., and Tack, A. J. 2020. The impact of elevated temperature and drought on the ecology and evolution of plant-soil microbe interactions. J. Ecol. 108:337-352.

Roberts, L. D., Souza, A. L., Gerszten, R. E., Clish, C. B. 2012. Targeted metabolomics. Curr. Protoc. Mol. Boil. 98:30.2.1-30.2.24.

Sakaigaichi, T., Tsuchida, H., Adachi, K., Hattori, T., Tarumoto, Y., Tanaka, M., Hayano, M., Sakagami, J. I., and Irei, S. 2019. Phenological changes in the chlorophyll content and its fluorescence in field-grown sugarcane clones under over-wintering conditions. Sugar Tech 21:843-846.

Salam, W. H., Wilcox, H. G., Cagen, L. M., and Heimberg, M. 1989. Stimulation of hepatic cholesterol biosynthesis by fatty acids. Effects of oleate on cytoplasmic acetoacetyl-CoA thiolase, acetoacetyl-CoA synthetase and hydroxymethylglutaryl-CoA synthase. Biochem. J. 258: 563-568.

SkZ, A., Vardharajula, S., and Vurukonda, S. S. K. P. 2018. Transcriptomic profiling of maize (Zea mays L.) seedlings in response to Pseudomonas putida stain FBKV2 inoculation under drought stress. Ann. Microbiol. 68:331-349.

Sui, J., Qu, C., Yang, J., Zhang, W., and Ji, Y. 2019. Transcriptome changes in the phenylpropanoid pathway in senescing leaves of Toona sinensis. Acta Physiol. Plant. 41:126.

Sun, H., Li, Y., Feng, S., Zou, W., Guo, K., Fan, C., Si, S., and Peng, L. 2013. Analysis of five rice 4-coumarate:coenzyme A ligase enzyme activity and stress response for potential roles in lignin and flavonoid biosynthesis in rice. Biochem. Biophys. Res. Commun. 430: 1151-1156.

Sureshrao, K. S., Pradeeprao, K. T., Dnyanobarao, G. S., Agrawal, T., and Kotasthane, A. 2016. Root growth stimulation in rice (Oryza sativa L.) by seed bio-priming with Trichoderma sp. Appl. Biol. Res. 18:30-38.

Thomas, J., Kim, H. R., Rahmatallah, Y., Wiggins, G., Yang, Q., Singh, R., Glazko, G., and Mukherjee, A. 2019. RNA-seq reveals differentially expressed genes in rice (Oryza sativa) roots during interactions with plant-growth promoting bacteria, Azospirillum brasilense. PLoS One 14: e0217309.

Toju, H., Peay, K. G., Yamamichi, M., Narisawa, K., Hiruma, K., Naito, K. Fukuda, S., Ushio, M., Nakaoka, S., Onoda, Y., Yoshida, K., Schlaeppi, K., Bai, Y., Sugiura, R., Ichihashi, Y., Minamisawa, K., and Kiers, E. T. 2018. Core microbiomes for sustainable agroecosystems. Nat. Plants 4: 247-257.

Trapnell, C., Roberts, A., Goff, L., Pertea, G., Kim, D., Kelley, D. R., Pimentel, H., Salzberg, S. L., Rinn, J. L., and Pachter, L. 2012. Differential gene and transcript expression analysis of RNA-seq experiments with TopHat and Cufflinks. Nat. Protoc. 7:562-578.

Trapnell, C., Williams, B. A., Pertea, G., Mortazavi, A., Kwan, G., van Baren, M. J., Salzberg, S. L., Wold, B. J., and Pachter, L. 2010. Transcript assembly and quantification by RNA-seq reveals unannotated transcripts and isoform switching during cell differentiation. Nat Biotechnol. 28:511-515.

Wang, H., Liu, Z., Xu, W., and Wang, Z. 2019. Determination of growthpromoting ability of several strains to rice. Jiangsu Agr. Sci. 47:94-99.

Wang, Z., Piao, Y., Zhang, F., Hu, Y., Zeng, J., and Nan, J. 2019a. Promoting effects on watermelon and fermentation optimization of Plantibacter sp. WZW03. J. Plant Growth Regul.

Wang, Z., Wang, C., You, Y., Xu, W., Lv, Z., Liu, Z., Chen, W., Shi, Y., and Wang, J. 2019b. Response of Pseudomonas fluorescens to dimethyl phthalate. Ecotoxicol. Environ. Saf. 167:36-43.

Whalley, W. R., Binley, A., Watts, C. W., Shanahan, P., Dodd, I. C., Ober, E. S., Ashton, R. W., Webster, C. P., White, R. P., and Hawkesford, M. J. 2017. Methods to estimate changes in soil water for phenotyping root activity in the field. Plant Soil 415:407-422.

Xia, J., Psychogios, N., Young, N., and Wishart, D. S. 2009. MetaboAnalyst: A web server for metabolomic data analysis and interpretation. Nucleic Acids Res. 37:W652-W660.

Xoca-Orozco, L. Á., Aguilera-Aguirre, S., Vega-Arreguín, J., AcevedoHernández, G., Tovar-Pérez, E., Stoll, A., Herrera-Estrella, L., and ChacónLópez, A. 2019. Activation of the phenylpropanoid biosynthesis pathway reveals a novel action mechanism of the elicitor effect of chitosan on avocado fruit epicarp. Food Res. Int. 121:586-592.

Yoshihara, S., Yamamoto, K., Nakajima, Y., Takeda, S., Kurahashi, K., and Tokumoto, H. 2019. Absorption of zinc ions dissolved from zinc oxide nanoparticles in the tobacco callus improves plant productivity. Plant Cell Tiss. Org. 138:377-385.

Zhu, X. F., Wang, Z. W., Wan, J. X., Sun, Y., Wu, Y. R., Li, G. X., Shen, R. F., and Zheng, S. J. 2015. Pectin enhances rice (Oryza sativa) root phosphorus remobilization. J. Exp. Bot. 66:1017-1024. 